\title{
Magnetic Propulsion of Self-Assembled Colloidal Carpets: Efficient Cargo Transport via a Conveyor-Belt Effect
}

\author{
Fernando Martinez-Pedrero ${ }^{1}$ and Pietro Tierno ${ }^{1,2, *}$ \\ ${ }^{1}$ Estructura i Constituents de la Matèria, Universitat de Barcelona, 08028 Barcelona, Spain \\ ${ }^{2}$ Institut de Nanociència i Nanotecnologia, IN ${ }^{2} U B$, Universitat de Barcelona, 08028 Barcelona, Spain
}

(Received 1 April 2015; revised manuscript received 20 April 2015; published 29 May 2015)

\begin{abstract}
We demonstrate a general method to assemble and propel highly maneuverable colloidal carpets which can be steered via remote control in any direction of the plane. These colloidal micropropellers are composed by an ensemble of spinning rotors and can be readily used to entrap, transport, and release biological cargos on command via a hydrodynamic conveyor-belt effect. An efficient control of the cargo transportation combined with remarkable "healing" ability to surpass obstacles demonstrate a great potential towards development of multifunctional smart devices at the microscale.
\end{abstract}

DOI: 10.1103/PhysRevApplied.3.051003

Dynamic self-assembly processes are widespread in physical systems and occur under out-of-equilibrium conditions when, for example, a driving field supplies energy and sustains a complex and otherwise unstable structure [1]. This powerful technique enables self-assembled systems to adapt to their environment or perform functional and sometimes programmable tasks. Examples at the microscale are disparate, including coherent patterns from an ensemble of driven [2-5] or active [6-9] systems.

Time-dependent magnetic fields applied to polarizable particles represent a convenient way to assemble and propel microscopic matter in a fluid medium since these fields do not affect the dispersing medium or alter biological systems unless these systems contain magnetic parts. The fabrication of magnetically driven artificial propellers is a research field of growing interest due to its direct application in biomedicine [10-12], targeted drug delivery [13], and microfluidics [14]. In addition, practical applications often require the use of micromachines capable of loading or unloading a defined cargo on command or to transport the cargo in a defined place of a microfluidic platform or a biological network. In this context, magnetic prototypes of various forms and shapes have been realized so far by using DNA-linked magnetic colloidal particles [15,16], helical structures [17,18], or other types of hybrid systems [19-21]. Besides a few recent examples [16,22], propelling structures based on pure dynamic self-assembly where cooperative interactions between the composing units lead to coherent motion of the whole system are still scarce. By applying suitable magnetic manipulation techniques, we experimentally demonstrate a way to manipulate and propel a large ensemble of microscopic particles assembled into highly ordered and maneuverable two-dimensional carpets via time-averaged dipolar forces. These carpets can be

ptierno@ub.edu assembled or disassembled at will, rotated or transported in any direction of the plane via magnetic control. Further, we show the possibility to entrap, transport, and release micro-objects such as biological cells or colloidal cargos in a fluidic environment, by using the hydrodynamic conveyor belt generated by the moving structure. These magnetic carpets, thus, represent an alternative class of propelling prototypes based on dynamic self-assembly.

As building blocks for the colloidal carpets, we use $2.8-\mu \mathrm{m}$-size monodisperse paramagnetic colloids (Dynabeads M-270, Invitrogen) composed by a polymer matrix and evenly doped with superparamagnetic grains (density $\rho=1.3 \mathrm{~g} \mathrm{~cm}^{-3}$ ). The original aqueous suspension of the particles is diluted with high-deionized water (18.2 $\mathrm{M} \Omega \mathrm{cm}$, MilliQ system) at a concentration of approximately $1.4 \times 10^{8}$ beads $/ \mathrm{ml}$. To prevent convection effects, the colloidal suspension is sandwiched between a glass slide (Corning Incorporated) and a microscope coverslip (Agar Scientific), both separated by a double-faced adhesive tape. As colloidal cargos, we use either budding yeast cells (Saccharomyces Cerevisiae) or silica-dioxide microspheres ( $5 \mu \mathrm{m}$ size, density $\rho=2.1 \mathrm{~g} \mathrm{~cm}^{-3}$ ) obtained from Sigma Aldrich. The measurement cell is placed in the center of two orthogonal pairs of coils arranged on the stage of a light microscope (Eclipse Ni, Nikon) and aligned with a fifth coil under the sample cell. The coils are connected with a waveform generator (TGA1244, TTi) feeding power amplifiers (AMP-1800, AKIYAMA and BOP 20-10M, Kepco). The paramagnetic colloids can be easily magnetized by a relatively low external field $\boldsymbol{H}$ acquiring a dipole moment $\boldsymbol{m}=V \chi \boldsymbol{H}$ pointing along the field direction. Here, $V$ is the particle volume and $\chi=0.4$ the magnetic susceptibility under a static field. For a rotating field circularly polarized in the $(x, z)$ plane, with angular frequency $\omega$ and amplitude $H_{0}$, $\boldsymbol{H}=H_{0}\left[\cos (\omega t) \boldsymbol{e}_{x}-\sin (\omega t) \boldsymbol{e}_{z}\right]$, the particle dynamics become more complicated due to the presence of a finite magnetization relaxation time $\tau_{r}[23,24]$. The average 
(a)
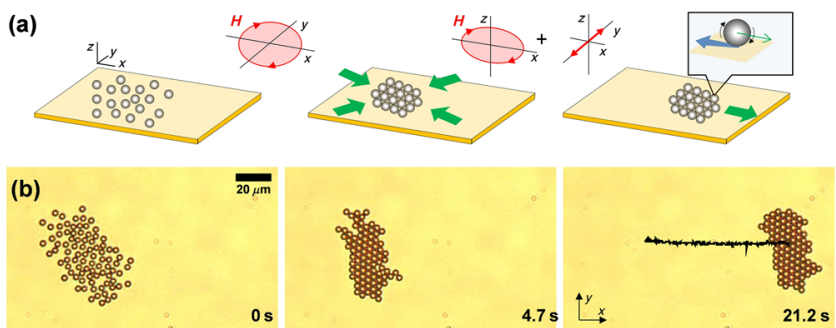

(c)

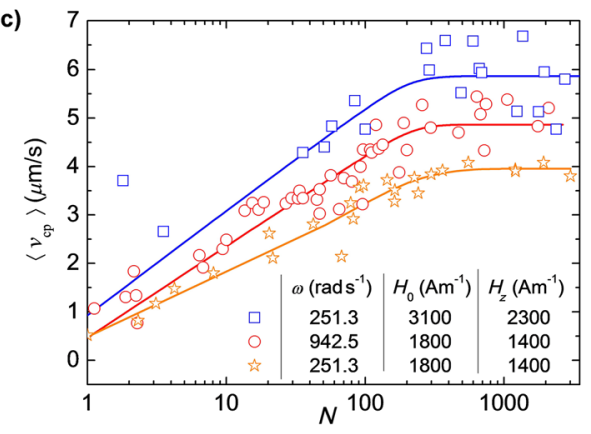

FIG. 1. Schematics showing the procedure to assemble (left middle) and propel (middle right) a colloidal carpet. Assembly is induced by a magnetic field rotating in the particle plane $(x, y)$, whereas propulsion is induced by a rotating field in the perpendicular plane $(x, z)$ plus an oscillating component along the $y$ axis. (b) Snapshots of a colloidal carpet assembled and propelled by an applied field with amplitudes $H_{0}=1800 \mathrm{~A} \mathrm{~m}^{-1}$, $H_{z}=1400 \mathrm{~A} \mathrm{~m}^{-1}$, and frequencies $\omega=2 \omega_{y}=942.5 \mathrm{rads}^{-1}$, (Movie S1 in Ref. [32]). (c) Semilog plot of the average speed $\left\langle v_{\text {cp }}\right\rangle$ versus number of particles $N$ composing the carpet at different field values and frequencies (here, $\omega_{y}=\omega / 2$ ).

magnetic torque applied to the particle can be calculated as $\boldsymbol{T}_{m}=\mu_{0}\langle\boldsymbol{m} \times \boldsymbol{H}\rangle$, where $\mu_{0}=4 \pi \times 10^{-7} \mathrm{H} \mathrm{m}^{-1}$ and $\langle\ldots\rangle$ denotes a time average. Solving the relaxation equation for the magnetic moment [25], we find that $\boldsymbol{T}_{m}=$ $\left[\mu_{0} V \chi H_{0}^{2} \tau_{r} \omega /\left(1+\tau_{r}^{2} \omega^{2}\right)\right] \boldsymbol{e}_{y}$, where $\tau_{r} \sim 10^{-4}$ s from independent measurements. The applied torque forces the particle to rotate at an average angular velocity $\Omega$ in the fluid medium. Upon balancing $\boldsymbol{T}_{m}$ with the viscous torque arising from the rotation in the medium $\boldsymbol{T}_{v}=-8 \pi \eta \Omega a^{3}$, the average rotational speed reads as $\langle\Omega\rangle=\mu_{0} H_{0}^{2} \chi \tau_{r} \omega /$ $6 \eta\left(1+\tau_{r}^{2} \omega^{2}\right)$. Here, $\eta=10^{-3}$ Pas denotes the dynamic viscosity of water. A pair of rotors also interacts via dipolar forces. The interaction potential between two equal dipoles $(i, j)$ at a distance $\boldsymbol{r}$ away is given by $U_{d d}=$ $\mu_{0} / 4 \pi\left\{\left(\boldsymbol{m}_{i} \cdot \boldsymbol{m}_{j} / r^{3}\right)-\left[3\left(\boldsymbol{m}_{i} \cdot \boldsymbol{r}\right)\left(\boldsymbol{m}_{j} \cdot \boldsymbol{r}\right) / r^{5}\right]\right\}$ and is maximally attractive (repulsive) for particles with magnetic moments parallel (normal) to $\boldsymbol{r}$. Time averaging $U_{d d}$ for a rotating magnetic field in the $(x, y)$ plane gives an effective attractive potential in this plane $\left\langle U_{d d}\right\rangle=-\left[\mu_{0} m^{2} /\right.$ $\left.8 \pi(x+y)^{3}\right]$. This interaction potential can be, thus, used to magnetically assemble highly ordered $2 \mathrm{D}$ particle monolayers [26,27].

Figure 1(a) shows the general procedure to realize and propel a magnetic carpet. First, compact clusters are assembled by applying an external field circularly polarized in the $(x, y)$ plane, $\boldsymbol{H} \equiv H_{0}\left[\cos (\omega t) \boldsymbol{e}_{x}-\sin (\omega t) \boldsymbol{e}_{y}\right]$. The applied field induces attractive dipolar interactions, and the particles rapidly assemble into a close-packed cluster free of defects or vacancies. The colloidal clusters are only stable in the presence of the driving field, while they immediately disintegrate due to thermal fluctuations once the applied field is switched off. As reported in previous works on assembly of "magnetic holes" [28] or Janus colloids [29], we observe that when the cluster is formed, it continues to rotate but at a smaller angular frequency than that imposed by the driving field. The cluster rotation arises from an unbalanced viscous shear force experienced by the torque particles located at the edge of the cluster $[29,30]$. Once the carpets are formed, propulsion is obtained by applying a rotating field in the $(x, z)$ plane plus an additional component oscillating with angular frequency $\omega_{y}$ along the perpendicular direction $(y)$. The total field is given by $\boldsymbol{H} \equiv H_{0}\left[\cos (\omega t) \boldsymbol{e}_{x}+\sin \left(\omega_{y} t\right) \boldsymbol{e}_{y}-\frac{H_{z}}{H_{0}} \sin (\omega t) \boldsymbol{e}_{z}\right]$, as depicted in the right part of Fig. 1(a). In most of the experiments reported here, we use $H_{0} \equiv H_{x}=H_{y}$ and $\omega_{y}=\omega / 2$. The rotating field in the $(x, z)$ plane exerts a torque on the individual particles, forcing them to rotate close to the glass substrate. The particles acquire a net translational motion with an average speed $\left\langle v_{x}\right\rangle \sim \Omega a$, due to the hydrodynamic coupling with the substrate [31], making possible the propulsion of the whole carpet. The $H_{y}$ component helps to keep the structure compact during motion, avoiding lateral separation of the constituent particles. The choice of $\omega_{y}=\omega / 2$ avoids the carpet rotation which, otherwise, would occur by simply setting $\omega_{y}=\omega$. Figure 1 (b) shows a carpet propelling with an average speed of $\left\langle v_{\mathrm{cp}}\right\rangle=$ $2.7 \mu \mathrm{m} / \mathrm{s}$ along the $x$ direction, keeping its structure intact. During motion, the carpet trajectory is quite stable, presenting negligible displacements in the perpendicular direction. In contrast to the assembly stage, the rotating field in the $(x, z)$ plane is now strongly elliptically polarized $\left(H_{0} \gg H_{z}\right)$ in order to maintain the structure confined to two dimensions.

Although the speed acquired by an individual particle (rotor) moving close to the substrate is relatively slow, for an ensemble of rotors forming the carpet the effect becomes cooperative resulting in a faster translational motion. In Fig. 1(c), this effect is reflected by measuring the average propulsion speed $\left\langle v_{\mathrm{cp}}\right\rangle$ as a function of the number of rotors $N$ for a series of carpets having approximately a spherical shape. The continuous dashed lines are averaged curves from the experimental data showing the general trend. While $\left\langle v_{\text {cp }}\right\rangle$ initially rapidly increases with $N$, it saturates around $N \sim 300$, corresponding to a carpet area $S \sim 1800 \mu \mathrm{m}^{2}$. Beyond this value, the rotors composing the colloidal structure start to be far away, and the cooperative effect reaches its maximum efficiency. Further, we observe that at parity of frequency, increasing the field amplitude $H_{0}$ 
[orange stars and blue squares in Fig. 1(c)] raises the average speed since the magnetic particles are subjected to higher magnetic torque and acquire a faster rotational motion. On the other hand, the propulsion speed can be also increased via the driving frequency [orange stars and red circles in Fig. 1(c)], although this effect is less pronounced.

The maneuverability of our colloidal structures is shown in Fig. 2(a), where the carpet is dynamically guided, respectively, towards the left, south, and north of the observation area. As shown in Movie S2a, these turns are achieved by temporarily stopping the propulsion $\left(H_{z}=0\right)$ and inducing a rotational motion of the carpet by setting $\omega_{y}=\omega=942.5 \mathrm{rad} \mathrm{s}^{-1}$. The change in direction is then obtained by restoring the field but exchanging the phases and frequencies of $H_{x}$ and $H_{y}$. In Figs. 2(b) and 2(c), we explore the stability of the propelling carpets by forcing them to move against an immobile obstacle, a 5- $\mu \mathrm{m}$ silica particle which was permanently attached to the substrate. Figure 2(b) (Movie S2b) shows the carpet dynamics without changing the applied field. In this case, the obstacle locally melts the moving crystal of rotors, but strong attractive dipolar interactions prevent its disaggregation, favoring recrystallization templated by the ordered particles surrounding the melted region. An alternative method to pass the obstacle is presented in Fig. 2(c) (Movie S2c). In this particular situation, we split the carpet into several pieces by setting temporary $H_{y}=0$ and decreasing the perpendicular field $H_{z}$. The absence of the $H_{y}$ component makes the structure less stable during its propulsion, inducing the lateral separation of the constituent particles preferentially along the crystalline axis as triggered by the obstacle. After crossing the obstacle, all pieces are reassembled by restoring the field parameters. We note that the splitting of the carpet into pieces leads to a final different colloidal structure. Nevertheless, we find that in both cases [Figs. 2(b) and 2(c)], the carpet is able to heal small wounds and adapt its shape to the most compact structure after crossing the obstacle.
Noninvasive approaches based on propulsive colloids to move and displace micro-objects have been achieved in the past by using elongated nanorods [33], anisotropic particles [34], and helical [35] or other composite structures $[36,37]$. Our self-assembled carpet allows us to manipulate and transport microscopic cargos over its extended area without requiring direct contact or chemical binding. This feature is demonstrated in Fig. 3(a) corresponding Movie S3a, where a carpet having an area of $S \sim 1200 \mu \mathrm{m}^{2}$ is used to transport one yeast cell along a $95-\mu \mathrm{m}$ straight path. First, as shown in MovieS3b and MovieS3c in [32], the loading of the cargo, here a biological cell, can be realized by direct entrapment (Movie S3b) or by lifting the cargo when the carpet moves or rotates close to it (Movie S3c). Once above the magnetic carpet, the microscopic cargo is transported by using the hydrodynamic flow generated by the rotating particles. As illustrated by the schematic of Fig. 3(b), this flow advects the colloidal carpet acting as a conveyor belt. In contrast to other techniques to displace micro-objects, we find that the cargo moves faster than the underlying structure. For instance, in the specific case of Fig. 3(a), the yeast cell travels at an average speed of $\left\langle v_{\mathrm{cg}}\right\rangle=3.5 \mu \mathrm{m} / \mathrm{s}$, twice larger than the speed of the carpet. This behavior is independent of the cargo nature, as shown in Fig. 3(d), where we found approximately the same ratio by employing $5-\mu \mathrm{m}$ silica particles. At parity of field strength, we observe that both $\left\langle v_{\mathrm{cg}}\right\rangle$ and $\left\langle v_{\mathrm{cp}}\right\rangle$ increase linearly with the frequency, and their ratio keeps constant. Increasing $\omega$ increases the rotational speed of the particles and, thus, the hydrodynamic flux above and below the carpet. While the colloidal cargo is free to be dragged by this flux, the colloidal carpet is slower since being closer to the surface it experiences a larger hydrodynamic drag [38]. Due to its high speed, the cargo will reach the edge and leave the carpet in a finite period of time. In order to transport the cargo for a longer distance, we manipulate the carpet inducing a $\pi$ rotation each time the cell reaches the

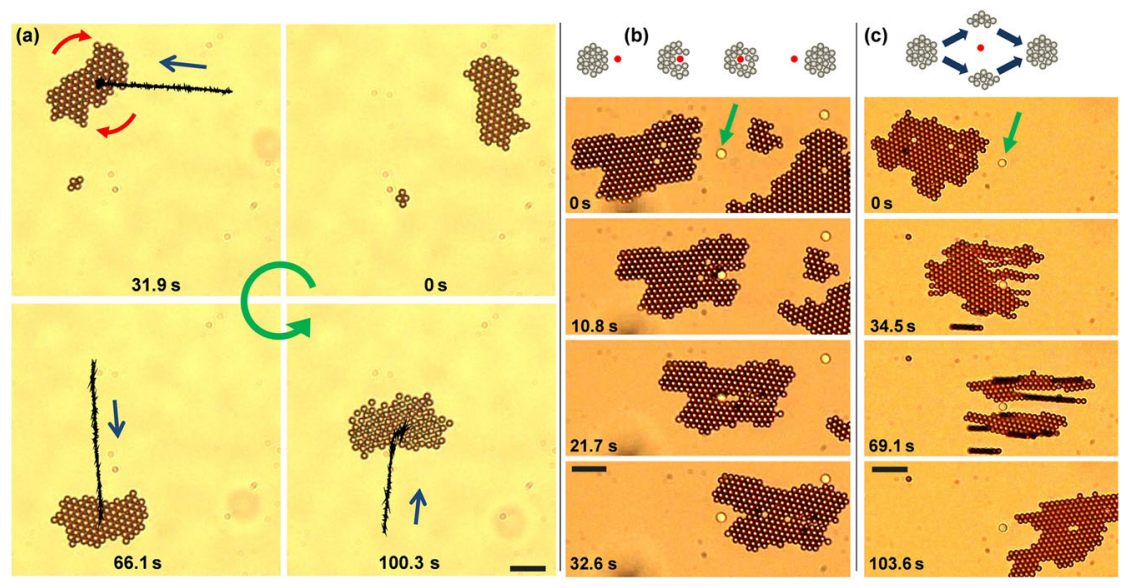

FIG. 2. (a) Snapshots in counterclockwise order, showing the guided motion of a colloidal carpet under dynamic magnetic fields with $H_{0}=2300 \mathrm{Am}^{-1}, H_{z}=1400 \mathrm{Am}^{-1}$ and $\omega=$ $2 \omega_{y}=942.5 \mathrm{rads}^{-1}$ (Movie S2a). Center-ofmass trajectory is superimposed as a black line. (b),(c) Top, schematic; bottom, sequence of images of colloidal carpets moving against a $5-\mu \mathrm{m}$ immobile silica particle. In (b), the field parameters remain constant $\left(H_{z}=\right.$ $1400 \mathrm{~A} \mathrm{~m}^{-1}, H_{0}=1800 \mathrm{~A} \mathrm{~m}^{-1}$ and frequencies $\omega=2 \omega_{y}=942.5 \mathrm{rads}^{-1}$, Movie S2b), while in (c), the field $H_{y}$ is set to zero, and $H_{z}$ decreases to $H_{z}=560 \mathrm{~A} \mathrm{~m}^{-1}$ after $t=26 \mathrm{~s}$ (Movie S2c). Later $(t=92.8 \mathrm{~s})$, the $H_{y}$ field is restored, and the carpet forms again. The scale bars in all images are $20 \mu \mathrm{m}$. 


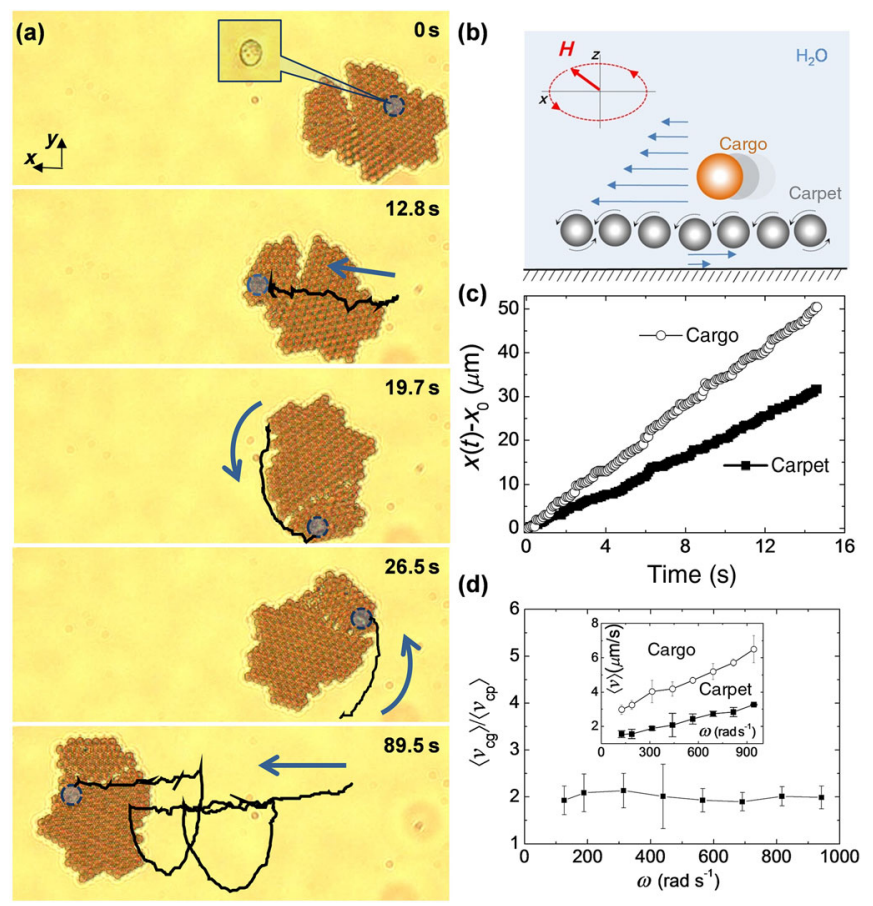

FIG. 3. (a) Sequence of images showing the transport of one yeast cell by combining translation and rotation of the carpet (Movie S3a). The position of the tracked cell is superimposed as a black line. The applied field parameters are the same as in Fig. 2(a). (b) Schematic of the hydrodynamic conveyor belt generated by the carpet. (c) Plots of the distance versus time for the carpet (filled squares) and cargo (empty circles) for Fig. 3(a) from $t=26.5 \mathrm{~s}$ to $t=41.0 \mathrm{~s}$. (d) Average speed of a colloidal cargo $\left\langle v_{\text {cg }}\right\rangle$ scaled with the carpet speed $\left\langle v_{\text {cp }}\right\rangle$ as a function of $\omega$ $\left(H_{z}=1400 \mathrm{~A} \mathrm{~m}^{-1}, H_{0}=1800 \mathrm{~A} \mathrm{~m}^{-1}\right.$, and $\left.\omega=2 \omega_{y}\right)$. Inset shows $\left\langle v_{\mathrm{cp}}\right\rangle$ and $\left\langle v_{\mathrm{cg}}\right\rangle$ separately.

edge of the structure. In the particular case of Fig. 3(a), two such rotations are required to allow the yeast cell to cover the whole observation area in $89.5 \mathrm{~s}$.

The ability to assemble and transport colloidal carpets close to a confining surface creates many opportunities to integrate them in a microfluidic platform. As an illustrative example, in Fig. 4 we assemble and guide two colloidal carpets in a microfluidic environment. We realize a 4- $\mu \mathrm{m}-$ depth channel structure above a glass plate by using a wet-etching hydrofluoric acid technique. The structure is characterized by a network of circular compartments having $115 \mu \mathrm{m}$ diameters and connected by channels $20 \mu \mathrm{m}$ wide. Free paramagnetic colloids previously collected in one circular compartment are assembled into two separate clusters having sizes of 670 and $430 \mu \mathrm{m}^{2}$ by an external rotating field. After $t=63.3 \mathrm{~s}$, the rotating field in the perpendicular plane is used to propel and guide these colloidal carpets along the path connecting the two chambers. Once the second compartment is reached $(t=276.4 \mathrm{~s})$, switching off the applied field melts the carpets, dispersing the particles evenly within the chamber. Even if no cargo is transported during this operation, one can use the paramagnetic colloids directly as drug delivery vectors, since their surface can be easily functionalized with chemical agents in order to bind and transport functional molecules [39]. Besides the basic geometries shown in Fig. 4, circuits having more complex patterns can be equally explored by the propelling carpets by simply adjusting on the fly the orientation and direction of the driving field.

In conclusion, we develop a simple and versatile technique to magnetically assemble and propel highly reconfigurable colloidal carpets in all directions of the plane. Although we demonstrate this method with commercially available paramagnetic colloids, it can be easily extended to other types of recently developed particles with heterogeneous magnetic properties such as cubes [40], ellipsoids [41], Janus [42], or anisotropic [43] ones. As opposed to existing microscopic engines that are chemically powered or magnetically propelled, the mechanism of motion of our carpets is cooperative and based on the rectification of the hydrodynamic flow generated by each rotor close to the bounding wall.

We thank Ignacio Pagonabarraga Mora for stimulating discussions. The authors acknowledge support from the

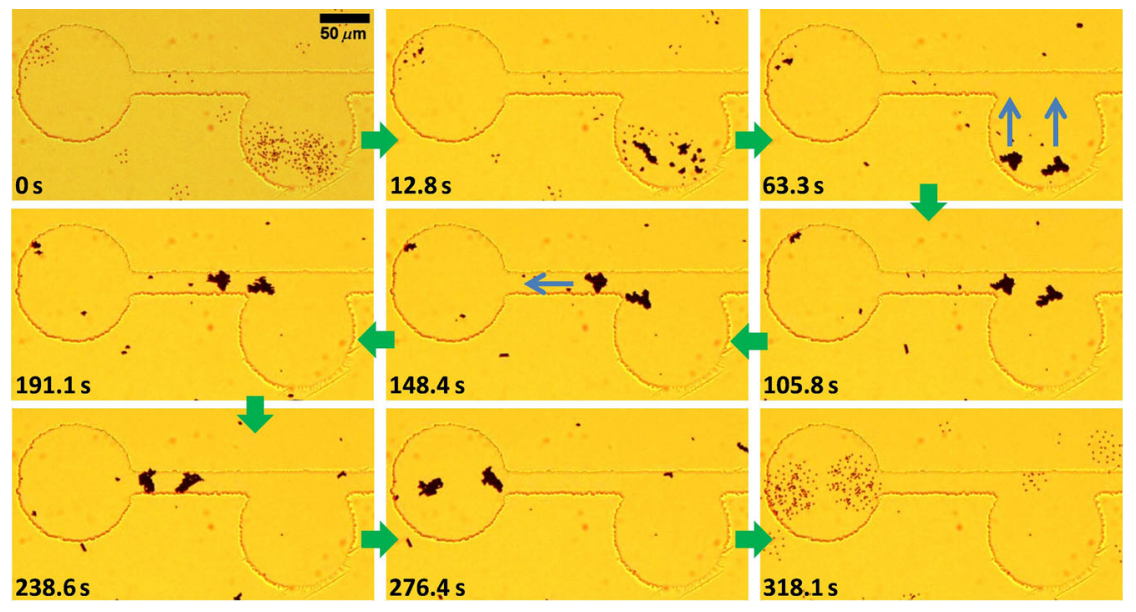

FIG. 4. Sequence of images showing the formation of two colloidal carpets from dispersed particles (top sequence) within a glass etched circular chamber (115 $\mu \mathrm{m}$ diameter). The carpets are later transported across thin microchannels $20 \mu \mathrm{m}$ width having (middle sequence), and finally the particles are redispersed by switching off the applied field 318.1 s later (Movie S4). The applied field parameters during transport are $H_{0}=$ $1900 \mathrm{~A} \mathrm{~m}^{-1}, H_{z}=980 \mathrm{Am}^{-1}, \omega_{z}=942.5 \mathrm{~Hz}$. The frequencies are $\omega_{x}=\omega_{z} / 2$ and $\omega_{y}=\omega_{z}$ $\left(\omega_{x}=\omega_{z}\right.$ and $\left.\omega_{y}=\omega_{z} / 2\right)$ when the carpets move upward (leftward). 
European Research Council Project No. 335040. P. T. acknowledges support from the "Ramon y Cajal" Program No. RYC-2011-07605, from Mineco (Grant No. FIS2013-41144-P), and AGAUR (Grant No. 2014SGR878).

[1] G. M. Whitesides and B. Grzybowski, Self-assembly at all scales, Science 295, 2418 (2002).

[2] B. A. Grzybowski, H. A. Stone, and G. M. Whitesides, Dynamic self-assembly of magnetized, millimetre-sized objects rotating at a liquidair interface, Nature (London) 405, 1033 (2000).

[3] M. V. Sapozhnikov, Y. V. Tolmachev, I. S. Aranson, and W.-K. Kwok, Dynamic Self-Assembly and Patterns in Electrostatically Driven Granular Media, Phys. Rev. Lett. 90, 114301 (2003).

[4] A. Snezhko and I. S. Aranson, Magnetic manipulation of self-assembled colloidal asters, Nat. Mater. 10, 698 (2011).

[5] J. V. I. Timonen, M. Latikka, L. Leibler, R. H. A. Ras, and O. Ikkala, Switchable static and dynamic self-assembly of magnetic droplets on superhydrophobic surfaces, Science 341, 253 (2013).

[6] W. Wang, W. Duan, A. Sen, and T. E. Mallouk, Catalytically powered dynamic assembly of rod-shaped nanomotors and passive tracer particles, Proc. Natl. Acad. Sci. U.S.A. 110, 17744 (2013).

[7] Ivo Buttinoni, Julian Bialké, Felix Kümmel, Hartmut Löwen, Clemens Bechinger, and Thomas Speck, Dynamical Clustering and Phase Separation in Suspensions of Self-Propelled Colloidal Particles, Phys. Rev. Lett. 110, 238301 (2013).

[8] Rodrigo Soto and Ramin Golestanian, Self-Assembly of Catalytically Active Colloidal Molecules: Tailoring Activity through Surface Chemistry, Phys. Rev. Lett. 112, 068301 (2014).

[9] Félix Ginot, Isaac Theurkauff, Demian Levis, Christophe Ybert, Lydéric Bocquet, Ludovic Berthier, and Cécile Cottin-Bizonne, Nonequilibrium Equation of State in Suspensions of Active Colloids, Phys. Rev. X 5, 011004 (2015).

[10] S. Sudo, S. Segawa, and T. Honda, Magnetic swimming mechanism in a viscous liquid, J. Intell. Mater. Syst. Struct. 17, 729 (2006).

[11] S. Guo, Q. Pan, and M. B. Khamesee, Development of a novel type of microrobot for biomedical application, Microsyst. Technol. 14, 307 (2008).

[12] B. J. Nelson, I. K. Kaliakatsos, and J. J. Abbott, Microrobots for minimally invasive medicine, Annu. Rev. Biomed. Eng. 12, 55 (2010).

[13] S. Kim, F. Qiu, S. Kim, A. Ghanbari, C. Moon, L. Zhang, B. J. Nelson, and H. Choi, Fabrication and characterization of magnetic microrobots for three-dimensional cell culture and targeted transportation, Adv. Mater. 25, 5863 (2013).

[14] S. Sanchez, A. A. Solovev, S. M. Harazim, and O. G. Schmidt, Microbots swimming in the flowing streams of microfluidic channels, J. Am. Chem. Soc. 133, 701 (2011).

[15] R. Dreyfus, J. Baudry, M. L. Roper, M. Fermigier, H. A. Stone, and J. Bibette, Microscopic artificial swimmers, Nature (London) 437, 862 (2005).
[16] P. Tierno, R. Golestanian, I. Pagonabarraga, and F. Sagués, Controlled Swimming in Confined Fluids of Magnetically Actuated Colloidal Rotors, Phys. Rev. Lett. 101, 218304 (2008).

[17] L. Zhang, J. J. Abbott, L. Dong, K. E. Peyer, B. E. Kratochvil, H. Zhang, C. Bergeles, and B. J. Nelson, Characterizing the swimming properties of artificial bacterial flagella, Nano Lett. 9, 3663 (2009).

[18] P. Fischer and A. Ghosh, Magnetically actuated propulsion at low Reynolds numbers: Towards nanoscale control, Nanoscale 3, 557 (2011).

[19] O. S. Pak, W. Gao, J. Wang, and E. Lauga, High-speed propulsion of flexible nanowire motors: Theory and experiments, Soft Matter 7, 8169 (2011).

[20] B. J. Williams, S. V. Anand, J. Rajagopalan, and M. T. A. Saif, A self-propelled biohybrid swimmer at low Reynolds number, Nat. Commun. 5, 3081 (2014).

[21] T. Qiu, T. -C. Lee, A. G. Mark, K. I. Morozov, R. Münster, O. Mierka, S. Turek, A. M. Leshansky, and P. Fischer, Swimming by reciprocal motion at low Reynolds number, Nat. Commun. 5, 5119 (2014).

[22] A. Snezhko, M. Belkin, I. S. Aranson, and W.-K. Kwok, Self-Assembled Magnetic Surface Swimmers, Phys. Rev. Lett. 102, 118103 (2009).

[23] P. Tierno, R. Muruganathan, and T. M. Fischer, Viscoelasticity of Dynamically Self-Assembled Paramagnetic Colloidal Clusters, Phys. Rev. Lett. 98, 028301 (2007).

[24] X. J. A. Janssen, A. J. Schellekens, K. van Ommering, L. J. van Ijzendoorn, and M. W. J. Prins, Controlled torque on superparamagnetic beads for functional biosensors, Biosens. Bioelectron. 24, 1937 (2009).

[25] A. Cebers and H. Kalis, Dynamics of superparamagnetic filaments with finite magnetic relaxation time, Eur. Phys. J. E 34, 30 (2011).

[26] N. Osterman, I. Poberaj, J. Dobnikar, D. Frenkel, P. Ziherl, and D. Babic, Field-Induced Self-Assembly of Suspended Colloidal Membranes, Phys. Rev. Lett. 103, 228301 (2009).

[27] J. Yan, S. C. Bae, and S. Granick, Colloidal superstructures programmed into magnetic janus particles, Adv. Mater. 27, 874 (2015).

[28] Jozef Černák and Geir Helgesen, Aggregation of magnetic holes in a rotating magnetic field, Phys. Rev. E 78, 061401 (2008).

[29] J. Yan, S. C. Bae, and S. Granick, Rotating crystals of magnetic Janus colloids, Soft Matter 11, 147 (2015).

[30] J. Schwarz-Linek, C. Valeriani, A. Cacciuto, M. E. Cates, D. Marenduzzo, A. N. Morozov, and W. C. K. Poon, Phase separation and rotor self-assembly in active particle suspensions, Proc. Natl. Acad. Sci. U.S.A. 109, 4052 (2012).

[31] A. J. Goldman, R. G. Cox, and H. Brenner, Slow viscous motion of a sphere parallel to a plane wall-i motion through a quiescent fluid, Chem. Eng. Sci. 22, 637 (1967).

[32] See the Supplemental Material at http://link.aps.org/ supplemental/10.1103/PhysRevApplied.3.051003 for supplemental videos.

[33] T. Petit, L. Zhang, K. E. Peyer, B. E. Kratochvil, and B. J. Nelson, Selective trapping and manipulation of microscale 
objects using mobile microvortices, Nano Lett. 12, 156 (2012).

[34] J. Palacci, S. Sacanna, A. Vatchinsky, P. M. Chaikin, and D. J. Pine, Photoactivated colloidal dockers for cargo transportation, J. Am. Chem. Soc. 135, 15978 (2013).

[35] S. Tottori, L. Zhang, F. Qiu, K. K. Krawczyk, A. FrancoObregón, and B.J. Nelson, Magnetic helical micromachines: Fabrication, controlled swimming, and cargo transport, Adv. Mater. 24, 811 (2012).

[36] S. Sundararajan, P. E. Lammert, A. W. Zudans, V. H. Crespi, and A. Sen, Catalytic motors for transport of colloidal cargo, Nano Lett. 8, 1271 (2008).

[37] M. S. Sakar, E. B. Steager, D. H. Kim, M. J. Kim, G. J. Pappas, and V. Kumar, Single cell manipulation using ferromagnetic composite microtransporters, Appl. Phys. Lett. 96, 043705 (2010).
[38] W. B. Russel, D. A. Saville, and W. R. Schowalter, Colloidal Dispersions (Cambridge University Press, Cambridge, England, 1991).

[39] L. Yao and S. Xu, Force-induced remnant magnetization spectroscopy for specific magnetic imaging of molecules, Angew. Chem., Int. Ed. Engl. 50, 4407 (2011).

[40] S. Sacanna, L. Rossi, and D. J. Pine, Magnetic click colloidal assembly, J. Am. Chem. Soc. 134, 6112 (2012).

[41] O. Güell, F. Sagués, and P. Tierno, Magnetically driven janus micro-ellipsoids realized via asymmetric gathering of the magnetic charge, Adv. Mater. 23, 3674 (2011).

[42] I. Sinn, P. Kinnunen, S. N. Pei, R. Clarke, B. H. McNaughton, and R. Kopelman, Magnetically uniform and tunable Janus particles, Appl. Phys. Lett. 98, 024101 (2011).

[43] D. Zerrouki, J. Baudry, D. Pine, P. Chaikin, and J. Bibette, Chiral colloidal clusters, Nature (London) 455, 380 (2008). 